\title{
Modulation of Crystallinity and Optical Properties in Composite Materials Combining Iron Oxide Nanoparticles and Dye- Containing Covalent Organic Frameworks
}

\author{
Roberto Sánchez-Naya ${ }^{a, b}$ (D) \\ Vladimir Stepanenko ${ }^{a}$ (i) \\ Karl Mandel *c,d (i) \\ Florian Beuerle*a,b (i) \\ a Julius-Maximilians-Universität Würzburg, Institut für Organische Chemie, Am \\ Hubland, 97074 Würzburg, Germany \\ b Julius-Maximilians-Universität Würzburg, Center for Nanosystems Chemistry (CNC), \\ Theodor-Boveri-Weg, 97074 Würzburg, Germany \\ 'Friedrich-Alexander-Universität Erlangen-Nürnberg, Department für Chemie und \\ Pharmazie, Anorganische Chemie, Egerlandstraße 1, 91058 Erlangen, Germany \\ ${ }^{d}$ Fraunhofer-Institut für Silicatforschung (ISC), Neunerplatz 2, 97082 Würzburg, \\ Germany \\ karl.mandel@fau.de; florian.beuerle@uni-wuerzburg.de \\ In loving memory of Dr. Ángela Valentín.
}

Received: 05.11.2020

Accepted after revision: 14.12 .2020

DOI: 10.1055/s-0040-1722655; Art ID: om-20-0040oa

License terms: (c)

C 2021. The Author(s). This is an open access article published by Thieme under the terms of the Creative Commons Attribution-NonDerivative-NonCommercial License permitting copying and reproduction so long as the original work is given appropriate credit. Contents may not be used for commercial purposes, or adapted, remixed, transformed or built upon. (https://creativecommons.org/licenses/by-nc-nd/4.0/)

Abstract Two series of organic-inorganic composite materials were synthesized through solvothermal imine condensation between diketopyrrolopyrrole dialdehyde DPP-1 and 5,10,15,20-tetrakis(4aminophenyl)porphyrin (TAPP) in the presence of varying amounts of either amino- or carboxy-functionalized superparamagnetic iron oxide nanoparticles (FeO). Whereas high FeO loading induced crosslinking of the inorganic nanoparticles by amorphous imine polymers, a lower $\mathbf{F e O}$ content resulted in the formation of crystalline covalent organic framework domains. All hybrid materials were analyzed by magnetization measurements, powder X-ray diffraction, electron microscopy, IR, and UV/Vis absorption spectroscopy. Crystallinity, chromophore stacking, and visible absorption features are directly correlated to the mass fraction of the components, thus allowing for a fine-tuning of materials properties.

Key words Covalent organic frameworks, diketopyrrolopyrroles, porphyrins, iron oxide nanoparticles, hybrid materials, superparamagnetism

\section{Introduction}

Organic-inorganic hybrid materials ${ }^{1}$ can combine highly variable properties of diverse components into multifunctional composites. In nature, the complex hierarchical assembly of brittle minerals and organic molecules results in biomaterials with exceptional fracture resistance, ${ }^{2}$ which

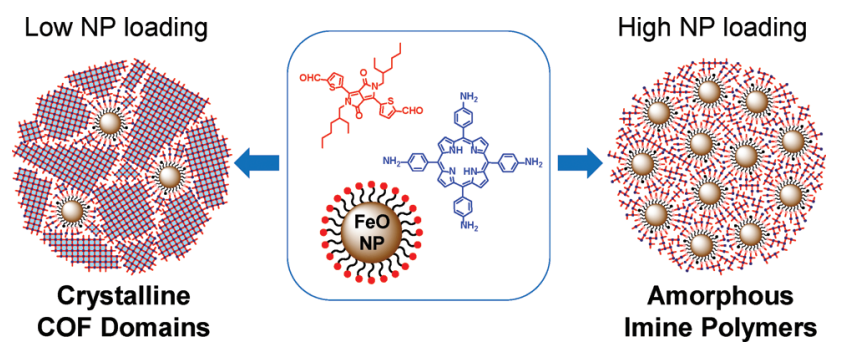

also inspired the design of biomimetic materials. ${ }^{3}$ Intriguingly, orthogonal functions that are typically hard to achieve in single-phase materials, e.g. luminescence ${ }^{4}$ and magnetism, can be successfully merged in artificial hybrid materials. In this regard, we recently succeeded in the combination of magnetic iron oxide nanoparticles and luminescent lanthanoid metal-organic frameworks into nanocomposites for switching isotropic and anisotropic optical properties, ${ }^{5}$ white magnetism, ${ }^{6}$ shear stress detection, ${ }^{7}$ or ratiometric water sensing. ${ }^{8}$

As another class of porous materials, covalent organic frameworks (COFs) ${ }^{9}$ have emerged as porous crystalline materials with potential applications in gas storage, ${ }^{10}$ heterogeneous catalysis, ${ }^{11}$ or organic electronics. ${ }^{12}$ Aiming for dye-containing $\mathrm{COFs},{ }^{13}$ we recently incorporated semiconducting diketopyrrolopyrrole ${ }^{14}$ (DPP) moieties into imine-linked DPP-TAPP-COF ${ }^{15}$ and boronate esterlinked DPP2-HHTP-COF ${ }^{16}$ that show efficient light harvesting in the visible region ${ }^{15}$ or electric conductivity in crystalline pellets. ${ }^{16}$ COF-based composite materials have been developed for (photo)catalysis ${ }^{17}$ and hydrogen evolution $^{18}$ in water splitting. Up to date, only a few examples for the combination of COFs and magnetic particles are reported, which have been used as magnetically recoverable adsorbents ${ }^{19}$ to remove toxins from biological samples, ${ }^{20}$ for solid-phase extraction ${ }^{21}$ or the photothermal conversion of near-IR absorption. ${ }^{22}$ However, detailed structural investigations are still needed to get a better understanding of organic-inorganic interphases. In particular, systematic studies on the effect of nanoparticle concentration and surface functionalization on the crystallinity and nanoscale morphology of the organic COF domains will help to further optimize the functional properties of these hybrid systems. 


\section{Results and Discussion}

Here we report on the synthesis and characterization of hybrid materials that combine superparamagnetic iron oxide nanoparticles and dye-containing COFs. Organicinorganic composites were obtained by the direct growth of organic COF domains at the surface of amino- or carboxyfunctionalized nanoparticles. Crystallinity, magnetic, optical, and electronic properties for two series of composites with varying mass fractions between COF and nanoparticle domains were studied by powder X-ray diffraction (PXRD), diffuse reflectance spectroscopy, and vibrating sample magnetometer (VSM) measurements. Here, we demonstrate that both crystallinity and absorption features of the COF domains could be controlled by adjusting the ratio between nanoparticle seeds and organic components.

Superparamagnetic nanoparticles were synthesized based on a reported procedure. ${ }^{23}$ Surfactant-free particles were
We recently reported the formation of dye-containing two-dimensional (2D) DPP-TAPP-COF via solvothermal imine formation between 5,10,15,20-tetrakis(4-aminophenyl)porphyrin (TAPP) and diketopyrrolopyrrole dialdehyde DPP-1 at $120{ }^{\circ} \mathrm{C}$ in ${ }^{n} \mathrm{BuOH} /$ mesitylene $(3: 1) .{ }^{15}$ Assuming that both, $\mathbf{N H}_{\mathbf{2}}-\mathbf{F e O}$ and $\mathbf{C O O H}-\mathbf{F e O}$, can initially bind aldehyde or amino precursors via imine or amide linkages, respectively, we investigated the growth of crystalline COF domains in the presence of FeO particles. COF synthesis was performed under optimized conditions ${ }^{15}$ but with varying $\mathbf{F e O}$ loading to study the effect of seed/monomer ratio on materials properties (see Scheme 1 ). Thereby, we obtained two series of organic-inorganic composites $\mathbf{N H}_{\mathbf{2}}$-FeO@DPP-TAPP-COF/POP (POP for porous organic polymer) and COOH-FeO@DPP-TAPP-COF/POP by adding 0.7, 1.4, 6.8, 13, 27, and $42 \mathrm{wt} \%$ of FeO seeds in relation to a hypothetical quantitative formation of the organic polymers.

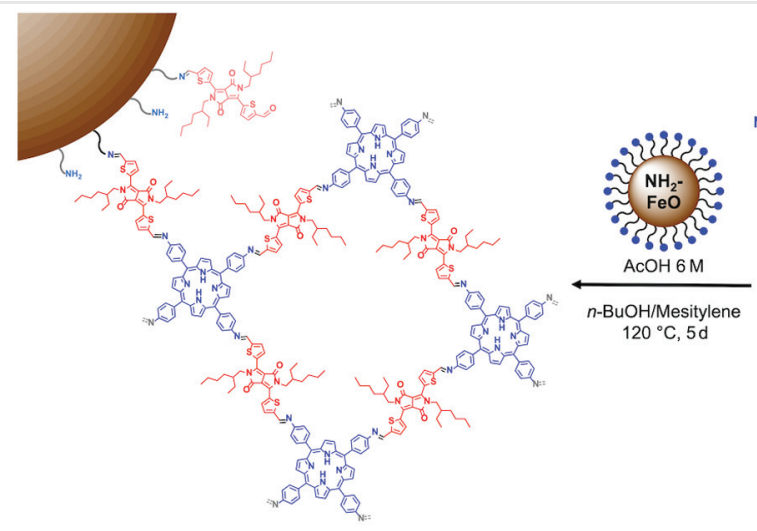

$\mathrm{NH}_{2}-\mathrm{FeO} @ D P P-T A P P-C O F(\mathrm{~N} 1-3)$ $\mathrm{NH}_{2}-\mathrm{FeO} @ D P P-T A P P-P O P(\mathrm{~N} 4-6)$

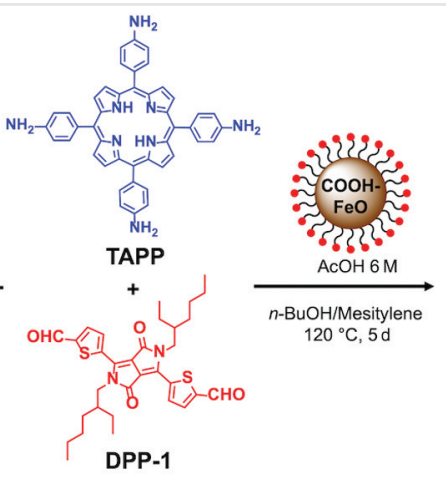

COOH-FeO@DPP-TAPP-COF (C1-4) COOH-FeO@DPP-TAPP-POP (C5-6)

Scheme 1 Synthesis of organic-inorganic composites FeO@DPP-TAPP-COF/POP via solvothermal COF synthesis in the presence of FeO seeds.

obtained by precipitation of a solution of $\mathrm{Fe}(\mathrm{III}) \mathrm{Cl}_{3}$ and $\mathrm{Fe}(\mathrm{II}) \mathrm{Cl}_{2}$ in water with aqueous ammonia solution. As shown in prior work, the nanoparticles obtained under these conditions cannot solely be assigned to be either pure magnetite $\left(\mathrm{Fe}_{3} \mathrm{O}_{4}\right)$ or maghemite $\left(\gamma-\mathrm{Fe}_{2} \mathrm{O}_{3}\right)$ but rather seem to be a complex mixture of both contributions and are therefore referred to as simply FeO in the following. Surface functionalization with either carboxylic acid (COOH-FeO) or amino groups $\left(\mathbf{N H}_{\mathbf{2}}-\mathbf{F e O}\right)$ was achieved by redispersion of unmodified particles in aqueous citric acid or covalent attachment of 3-aminopropyltriethoxysilane, respectively (see the Experimental Section for further details). Functionalized FeOs were separated by collection with an external magnet and centrifugation. Redispersion in ${ }^{t} \mathrm{BuOH}$ yielded stable organic dispersions (4 wt\%) for further functionalization.
For better readability, we introduce the nomenclature $\mathbf{N} \boldsymbol{x}$ and $\mathbf{C} \boldsymbol{x}$ ( $\boldsymbol{x}=1-6$ with increasing nanoparticle content) for the $\mathbf{N H}_{\mathbf{2}}$-FeO- or $\mathbf{C O O H}-\mathbf{F e O}$-based composites, respectively. Solvothermal reaction of DPP-1, TAPP, and $\mathbf{N H}_{\mathbf{2}}-\mathbf{F e O}$ or $\mathbf{C O O H}-\mathbf{F e O}$ in ${ }^{n} \mathrm{BuOH} /$ mesitylene (3:1) for 5 days resulted in precipitation of magnetizable composite materials (see Figure 1 for photographs of all composites under the effect of an external magnetic field). As expected however, magnetizability significantly decreased for composites X1-2 with lowest FeO content. Notwithstanding, separation from nonmagnetic side products and remaining starting materials with a simple magnet made purification by repetitive washing with anhydrous THF much easier for all composites, thus showcasing the potential of magnetically manipulable COF materials. 

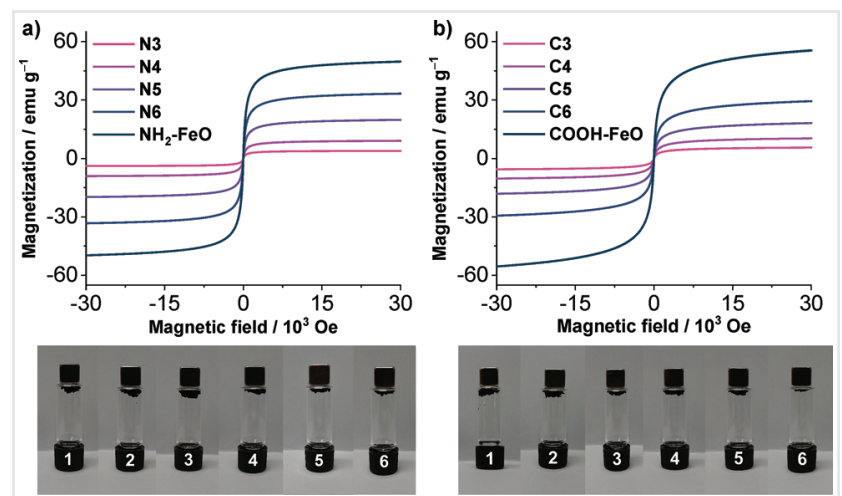

Figure 1 Saturation magnetization measurements for composites X3-6 and the pure FeO particles as reference and photographs of bulk samples of both N1-6 and C1-6 under the effect of an external magnetic field.

All materials were characterized by energy-dispersive Xray (EDX) spectroscopy, scanning electron microscopy (SEM), VSM measurements, Fourier-transform infrared (FT-IR) spectroscopy, PXRD, and diffuse reflectance spectroscopy to ultimately prove the formation of composites in all systems. In particular, we were interested in identifying and quantifying both inorganic and organic domains besides analysis of morphology, nanoscale structure, and optical properties for the integrated chromophores. Presence of DPP and TAPP moieties in all samples was confirmed by signals for $\mathrm{C}, \mathrm{O}, \mathrm{N}$, and $\mathrm{S}$ in EDX spectra (Figures S1 and S2). Whereas no Fe could be detected for scarcely loaded $\mathbf{N 1}$ and $\mathbf{C 1}$, increasing signals at $0.55(0), 0.7$ $(\mathrm{Fe})$, and $6.4(\mathrm{Fe}) \mathrm{KeV}$ indicated the growing proportion of $\mathbf{F e O}$ in both series of composites X2-6.

SEM revealed a granular morphology for all composites (Images S1-S12). The use of an energy-selective backscattered electron detector enhanced the chemical contrast and showed the homogeneous and increasingly dense distribution of $\mathbf{F e O}$ particles throughout the organic matrices for X3-6 (Images S13 and S14). For X1-2, no materials contrast was observed due to the very high dilution of the $\mathbf{F e O}$ nanoparticles in extended COF domains. However, the apparent magnetizability of these samples under the influence of an external magnetic field (see photographs in Figure 1) still indicates the incorporation of tiny amounts of nanoparticles. Magnetization for the composites was measured with a VSM. For pure $\mathbf{N H}_{\mathbf{2}}-\mathbf{F e O}$ and $\mathbf{C O O H}-\mathbf{F e O}$, a saturation magnetization of 50 and $56 \mathrm{emu} \mathrm{g}^{-1}$, respectively, was measured. Figure 1 shows the increment for the magnetization curves within the X3-6 series of composites, with saturation values of 3.8, 9.1, 19.8, and 33.3 emu $^{-1}$ for N3-6 and 5.6, 10.3, 18.1, and $29.4 \mathrm{emu} \mathrm{g}^{-1}$ for C3-6. For X1-2, the magnetic content in the composites was not sufficient for reliable VSM measurements. Based on previous investigations, we can state that the magnetic interaction of nanoparticles, when embedded together in a matrix, might change significantly, depending on their distance and/or geometrical arrangement to each other. The saturation magnetization however, i.e., the absolute magnetization that can be achieved with the particles or assemblies thereof, is not changed but rather reduced in magnetization per mass by the mass fraction of any nonmagnetic material that is added to the system. ${ }^{24}$ Under this assumption, we calculated a nanoparticle content of 8, 18, 40, and $67 \mathrm{wt} \%$ for N3-6 and 10, 19, 33, and 53 wt\% for C3-6 from these values, which are in good agreement with the added amounts of $\mathbf{F e O}$ particles, thus confirming the quantitative immobilization of the particles in the organic matrix (see Table S3 for more detailed information).

The nature of the organic cross-linkages was investigated by FT-IR spectroscopy. In all materials, the characteristic signature for DPP-TAPP-COF ${ }^{15}$ was observed (Figure S4). In particular, the absence of the aldehyde band for DPP-1 and the occurrence of a $\mathrm{C}=\mathrm{N}$ stretching mode at $1580 \mathrm{~cm}^{-1}$ confirmed the efficient formation of imine polymers in all composites. With increasing FeO content in both X1-6 series, a rather broad band arises with two characteristic maxima at 580 and $630 \mathrm{~cm}^{-1}$ corresponding to the $\mathrm{Fe}-\mathrm{O}$ stretching vibrations in the inorganic domains. Whereas FTIR confirmed the expected functional groups and linkages at the atomic scale, PXRD measurements probe the crystallinity and nanoscale arrangement (Figure 2). For N1-3 and C1-4 (Figures 2, S5, and S6), PXRD data confirmed the

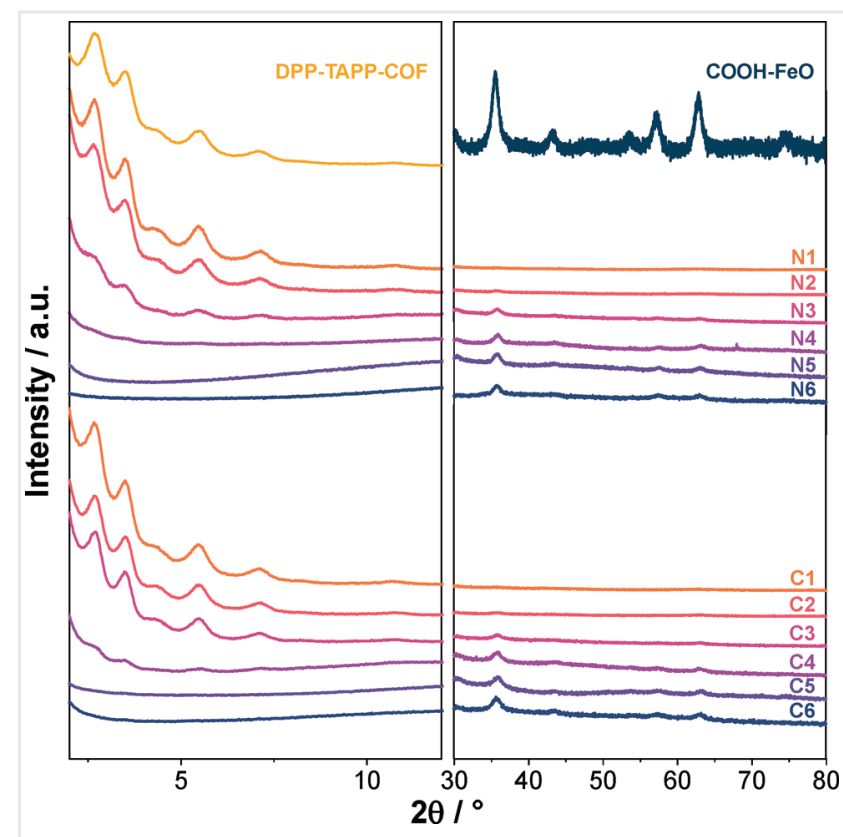

Figure 2 PXRD data for composites N1-6 and C1-6 and pure components DPP-TAPP-COF and $\mathrm{COOH}-\mathrm{FeO}$ as reference. 
formation of crystalline DPP-TAPP-COF domains with Bragg reflections centered at $2 \theta$ angles of $2.68^{\circ}, 3.51^{\circ}, 4.26^{\circ}, 5.49^{\circ}$, and $7.17^{\circ}$ that correspond to $110,020,120,220$, and 040 planes, respectively, for the monoclinic $C 2 / \mathrm{m}$ space group of the COF. ${ }^{15}$ With increasing FeO amount, the crystallinity of the COF domains decreased and only amorphous organic phases are observed for N4-6 and C5-6. In contrast, the corresponding reflexes for the inverse cubic spinel structure of $\mathrm{Fe}_{3} \mathrm{O}_{4}$ arose at $2 \theta$ values of $30.48^{\circ}, 35.85^{\circ}, 43.45^{\circ}, 53.89^{\circ}$, $57.44^{\circ}$, and $63.07^{\circ}$ corresponding to the $220,311,400,422$, 511 , and 440 planes, respectively. ${ }^{25}$

Based on these combined analytical data, we propose the following mechanism for composite formation (Figure 3a). Initially, organic precursors or smaller imine oligomers are covalently attached to the functionalized FeO surface, either via imine formation between aldehydes of DPP-1 and $\mathrm{NH}_{2}$-groups of $\mathbf{N H}_{2}-\mathbf{F e O}$ or amide formation between $\mathrm{NH}_{2}$-groups of TAPP and $\mathrm{COOH}$-groups of $\mathbf{C O O H}$ $\mathbf{F e O}$ for the $\mathbf{N x}$ and $\mathbf{C x}$ series, respectively. Due to structural constraints at the inorganic-organic interface, these seeds initiate rather amorphous imine polymerization in close proximity to the particle surface. In case of high FeO loading, these seeds are quickly cross-linked into composite materials without nanoscale ordering of the linking imine polymer network. With decreasing proportion of FeO however, there is enough time and organic feedstock to grow highly ordered COF domains in the periphery of the inorganic seeds, which are ultimately distributed within the crystalline matrix like "raisins in a cake" (Figure 3b).

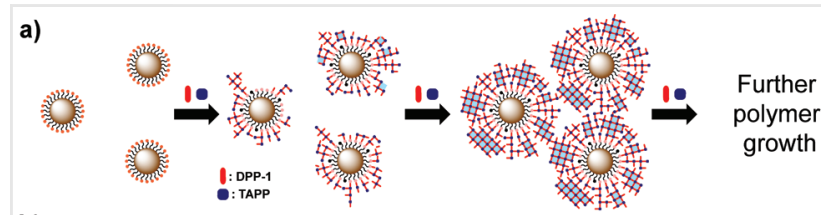

b)

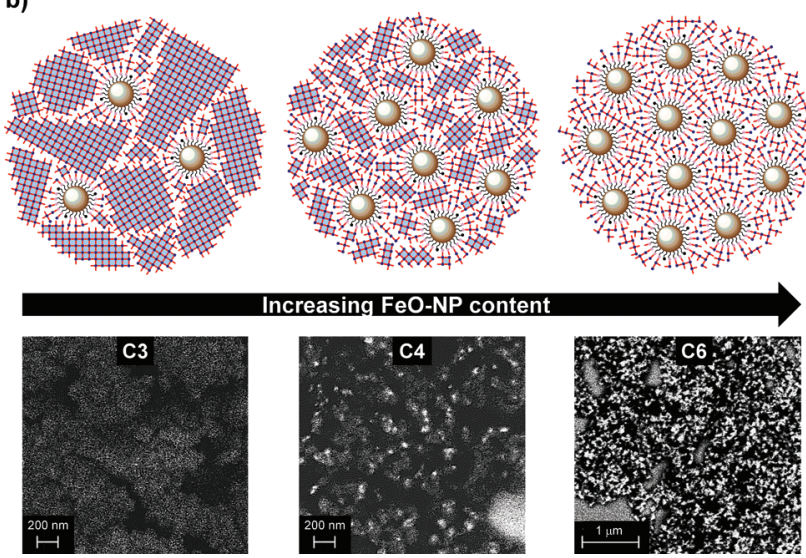

Figure 3 a) Proposed mechanism for composite formation and b) scanning electron microscopy images obtained with a back-scattered electron (BSE) detector indicating different degrees of FeO loading for selected samples of the $\mathbf{C} \boldsymbol{x}$ series.
Strikingly, the $\mathbf{C} \boldsymbol{x}$ series exhibited a higher crystallinity than the $\mathbf{N} \boldsymbol{x}$ series, as especially evidenced from PXRD for $\mathbf{C} 3$ and N3 with comparable FeO content but significantly different reflex intensities for the COF planes (Figure 2).

This ordering effect is most probably explained by the subtle differences in nucleation events at the particle surface. For $\mathbf{N H}_{\mathbf{2}}-\mathbf{F e O}$, the initial organic shell is most likely formed by linear DPP units, which are covalently attached via dynamic imine bonds. For $\mathbf{C O O H}-\mathbf{F e O}$ however, FT-IR spectra for the higher loaded composites C4-6 showed an incremental increase in intensity for the two amide signals at 1673 and $1580 \mathrm{~cm}^{-1}$ (Figure S3), thus suggesting that TAPP precursors are irreversibly attached via amide bonds to the particle surface. Therefore, we postulate that the primary attachment of the tetragonal porphyrin units to COOH-FeO might facilitate the formation of crystalline domains due to pronounced $\pi$-stacking of the porphyrins and the immediate possibility to extend the frameworks into three rather than just one direction (see Scheme 1 for a model of the initial organic shell). However, a catalytic effect of the acidic surface and the higher chemical stability of the amide linkages might also contribute to the higher crystallinity for the $\mathbf{C} \boldsymbol{x}$ series. Under the assumption that the added FeO particles quantitatively remain in the composites, we also obtained a slightly higher average yield of $68 \%$ compared to $57 \%$ for $\mathbf{C} \boldsymbol{x}$ and $\mathbf{N x}$, respectively (53\% for pure DPP-TAPP-COF, ${ }^{15}$ see the Supporting Information for further details). From these yields, we calculated FeO contents of 1, 3, 12, 21, 42, and 56 wt\% for N1-6 and 1, 2, 9, 17, 33, and 59 wt\% for C1-6, which are in good agreement with the values obtained from the VSM measurements. As demonstrated, the selection of composition can control the structure of the hybrid system and these structural properties ultimately do influence the optical properties of these hybrid materials.

As we have previously shown for the pure DPP-TAPPCOF, ${ }^{15}$ implementation of both DPP and porphyrin chromophores into a conjugated 2D imine polymer results in a significant shift of the maximum absorption peak due to planarization and pronounced stacking within the crystalline framework. Figure 4a shows UV/Vis absorption spectra for solid samples of DPP-1, TAPP, and DPP-TAPP-COF derived from diffuse reflectance measurements via the Kubelka-Munk function. For TAPP, the Soret band and three $\mathrm{Q}$ bands are observed at 430 and 525, 580, and $675 \mathrm{~nm}$, respectively. ${ }^{26}$ For DPP-1, one broad band around $600 \mathrm{~nm}$ is attributed to the DPP charge transfer transition. ${ }^{27}$ For a ground 1:2 mixture of TAPP and DPP-1, which complies with the composition of the COF, no peak shifts but rather a change in the relative ratio between the Soret and $\mathrm{Q}$ bands is observed, which is typically attributed to $\pi-\pi$ stacking of the porphyrin units in the solid state. ${ }^{28}$ For DPP-TAPP-COF however, the absorption maximum is shifted towards $693 \mathrm{~nm}$ and an almost complete attenuation of the 

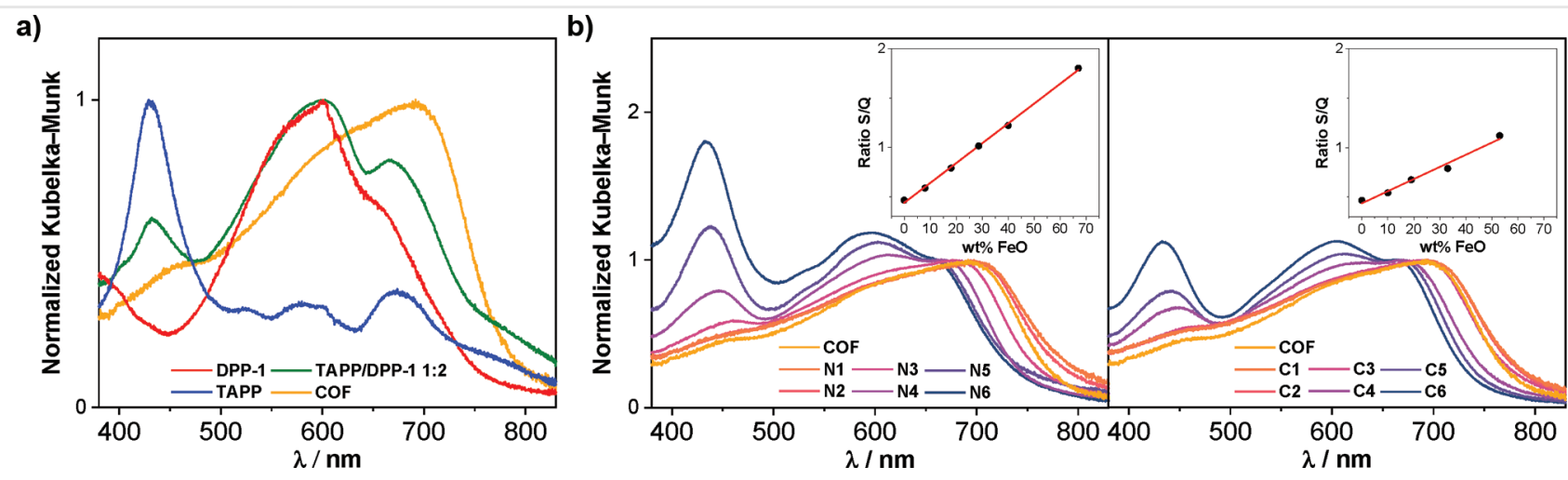

Figure 4 UV/Vis absorption spectra derived from diffuse reflectance measurements with the Kubelka-Munk function for a) solid samples of reference compounds DPP-TAPP-COF, TAPP, DPP-1, and a ground mixture of TAPP/DPP-1 with a 1:2 molar ratio (normalized to the global absorption maximum) and b) N1-6 and C1-6 composite series (normalized to the local absorption maximum with highest wavelength; the insets show the linear dependence of the Soret/Q band intensity ratio and mass fraction of $\mathrm{FeO}$ particles).

porphyrin Soret band is observed, which we previously attributed to both very good intralayer conjugation and interlayer aggregation within the 2D COF. The optical signature of DPP-TAPP copolymers should therefore serve as a sensitive probe for the nanoscale arrangement of the chromophores.

For the composite materials N1-2 and C1-3, nearly identical absorption spectra compared to the pure COF were observed (Figures 4a, b, and S8). The formation of extended and undisturbed DPP-TAPP-COF domains in these materials is further corroborated by the presence of distinct COF reflexes in PXRD data for these materials
(Figures 2, S5, and S6). Starting with $\mathbf{N 3}$ and $\mathbf{C 4}$, any further increase in $\mathbf{F e O}$ content is accompanied with the rise of two bands at around 610 and $445 \mathrm{~nm}$ and a hypsochromic shift of the low-energy maximum (Figures 4a, b, and S9). Here, the optical features are in much better accordance with the nonreacted 2:1 DPP-1/TAPP mixture, thus indicating the amorphous cross-linking of the particles as also seen in the PXRD data. Furthermore, the apparent trend in the relative intensity ratio between the Soret and $Q$ bands illustrates the influence of varying $\mathbf{F e O}$ content on the $\pi-\pi$ stacking and nanoscale arrangement of the TAPP moieties.

Table 1 Magnetization, mass fraction, and optical and electronic properties for composite materials N1-6 and C1-6

\begin{tabular}{|c|c|c|c|c|c|c|}
\hline Composite number & $\mathrm{FeO}$ added $^{\mathrm{a}}(\mathrm{wt} \%)$ & Magnetization $\left(\mathrm{emu} \mathrm{g}^{-1}\right.$ ) & $\mathrm{FeO}$ content (wt\%) & COF yield (\%) & S/Q ratio ${ }^{e}$ & $E_{\text {band gap }}(\mathrm{eV})$ \\
\hline N1 & 0.7 & Not measurable & $1.2^{\mathrm{c}}$ & 61 & 0.519 & 1.60 \\
\hline N2 & 1.4 & Not measurable ${ }^{\mathrm{b}}$ & $3.0^{c}$ & 50 & 0.530 & 1.62 \\
\hline N3 & 6.8 & 4 & $8.0^{\mathrm{d}}$ & 62 & 0.588 & 1.66 \\
\hline N4 & 13 & 9 & $18^{\mathrm{d}}$ & 61 & 0.790 & 1.69 \\
\hline N5 & 27 & 20 & $40^{d}$ & 56 & 1.223 & 1.72 \\
\hline \multirow[t]{2}{*}{ N6 } & \multirow[t]{2}{*}{42} & \multirow[t]{2}{*}{33} & $67^{d}$ & 47 & \multirow[t]{2}{*}{1.803} & \multirow[t]{2}{*}{1.70} \\
\hline & & & Average yield: & 56 & & \\
\hline $\mathrm{C} 1$ & 0.7 & Not measurable ${ }^{\mathrm{b}}$ & $1.0^{c}$ & 73 & 0.527 & 1.60 \\
\hline $\mathrm{C} 2$ & 1.4 & Not measurable & $2.3^{c}$ & 64 & 0.533 & 1.62 \\
\hline C3 & 6.8 & 6 & $10^{\mathrm{d}}$ & 72 & 0.543 & 1.64 \\
\hline $\mathrm{C} 4$ & 13 & 10 & $19^{d}$ & 69 & 0.675 & 1.67 \\
\hline C5 & 27 & 18 & $33^{d}$ & 74 & 0.789 & 1.70 \\
\hline \multirow[t]{2}{*}{ C6 } & \multirow[t]{2}{*}{42} & \multirow[t]{2}{*}{29} & $53^{d}$ & 58 & \multirow[t]{2}{*}{1.122} & \multirow[t]{2}{*}{1.72} \\
\hline & & & Average yield: & 68 & & \\
\hline
\end{tabular}

Related to hypothetical $100 \%$ COF formation.

bSaturation magnetization of less loaded composites $\mathbf{X} 1-\mathbf{2}$ could not be quantified due to very low magnetization.

${ }^{c}$ Calculated under the assumption that all added $\mathbf{F e O}$ remains in the composites.

${ }^{\mathrm{d} C}$ Calculated from VSM measurements.

eRelative ratio of intensity for the Soret and lowest energy $Q$ band of the porphyrin units. 
To quantify this effect, we normalized all spectra to the $Q$ band with the highest wavelength and plotted the relative intensity of the Soret band against the mass fraction of the FeO particles. Intriguingly, we obtained a linear relationship for both N2-6 and C3-6, thus indicating that the degree of $\pi$-stacking in the rather amorphous organic phases directly correlates to the nanoparticle content. Therefore, these linear regressions could be used to estimate the mass fraction and saturation magnetization of $\mathbf{F e O}$ domains directly from absorption data without the need for VSM measurements. Furthermore, the significantly flatter slope for $\mathbf{C} \boldsymbol{x}$ in comparison to $\mathbf{N} \boldsymbol{x}$ again demonstrates the superior nucleation capability of $\mathbf{C O O H}-\mathbf{F e O}$, as more ordered COF domains are formed in higher yields for identical nanoparticle loading.

Using a Tauc plot, we also estimated the band gaps for all composites from the absorption data for both series (Figures S10-S12 and Table 1). For the crystalline materials X1-3, one transition with band gaps between 1.6 and $1.7 \mathrm{eV}$ was observed, which is in very good agreement with the value of $1.64 \mathrm{eV}$ for the pure DPP-TAPP-COF. Interestingly, in the higher loaded composites $\mathbf{X 4 - 6}$, this band gap is shifted towards higher values of around $1.7 \mathrm{eV}$, with is in good agreement with values obtained for the ground 1:2 mixture of TAPP and DPP-1 as a model for the amorphous imine polymer phase. For the intermediate composites, we propose the simultaneous occurrence of two different organic phases, namely highly crystalline COF domains and ill-defined agglomerates of the chromophores for the amorphous imine polymers. Due to both more pronounced intramolecular $\pi-\pi$ stacking and better conjugation within the imine polymers, the crystalline COF phases possess a significantly lower band gap in comparison to the amorphous domains, thus highlighting the beneficial effect of a highly ordered chromophore arrangement in COF-based organic semiconductors. However, in both series, composites X1-2 with the lowest nanoparticle loading have an even smaller band gap as pure DPP-TAPP-COF (1.60 and $1.62 \mathrm{vs} .1 .64 \mathrm{eV}$ ). At this point, it is hard to tell if this effect is significant or just an artifact from the manual determination of the band gaps from the Tauc plots. However, it could well be that doping of the COF matrices with superparamagnetic $\mathbf{F e O}$ nanoparticles has an intrinsic influence on the photophysical properties of the semiconducting organic domains. An in-depth analysis of these effects will be the subject of future studies.

\section{Conclusions}

In conclusion, two series of composite materials $\mathbf{N H}_{2}$ Fe0@DPP-TAPP-COF/POP and COOH-FeO@DPP-TAPPCOF/POP were synthesized in solvothermal COF reactions of DPP and porphyrin monomers at the functionalized interface of $\mathbf{F e O}$ nanoparticles. SEM images and magnetization measurements revealed that the varying amounts of inorganic nanoparticles are homogeneously distributed within the polymeric organic matrix. Even for very low FeO loading, magnetizable samples were obtained, which simplifies purification and facilitates further manipulation by external magnetic fields. PXRD and UV/Vis absorption measurements revealed a gradual transition from crystalline DPP-TAPP-COF domains towards amorphous imine polymers with increasing $\mathbf{F e O}$ content. Thereby, crystallinity, nanoscale stacking, optical features, and band gaps of the semiconducting organic domains were precisely controlled by adjusting the mass fraction of the two components. Based upon this proof-of-concept study, we are now aiming for functional composites that combine magnetic and/or catalytically active inorganic nanoparticles with semiconducting organic polymers in a highly tunable manner.

\section{Experimental Section}

All solvents were purchased from Sigma Aldrich, Germany. ${ }^{n} \mathrm{BuOH}$ came anhydrous and packed under argon and mesitylene was degassed before use. $\mathrm{BaSO}_{4}$ as solid matrix for diffuse reflectance experiments was purchased from Alfa Aesar, Germany. Iron(III) chloride hexahydrate $\left(\mathrm{FeCl}_{3} \cdot 6 \mathrm{H}_{2} \mathrm{O}, 97.0 \%\right.$ purity) and iron(II) chloride tetrahydrate $\left(\mathrm{FeCl}_{2} \cdot 4 \mathrm{H}_{2} \mathrm{O}, 99.0 \%\right.$ purity) were purchased from Honeywell. Aqueous ammonia solution $\left(\mathrm{NH}_{3} 25 \mathrm{wt} \%\right.$ in $\left.\mathrm{H}_{2} \mathrm{O}\right)$ was purchased from Sigma-Aldrich. Citric acid monohydrate $\left(\mathrm{C}_{6} \mathrm{H}_{8} \mathrm{O}_{7} \mathrm{H}_{2} \mathrm{O}, 99.5 \%\right.$ purity) was provided from PanReac. 3Aminopropyltriethoxysilane $\left(\mathrm{C}_{9} \mathrm{H}_{23} \mathrm{NO}_{3} \mathrm{Si}, 98.0 \%\right.$ purity) was received from abcr. FT-IR spectroscopy was performed with a Jasco FT/IR-4600 spectrometer equipped with ATR-module. PXRD was conducted with a Bruker D8 Discover diffractometer in reflection mode with $\mathrm{Ni}$-filtered $K_{\alpha}$-radiation $(\lambda$ $=1.54060 \AA$ ) and a position-sensitive detector (LynxEye). The PXRD measurements of resulting materials were carried out on a silicon wafer and by applying a scan speed of $0.25 \mathrm{~s}$ per step and a $2 \theta$ angle increment of $0.02^{\circ}$. Diffuse reflectance measurements were performed with a Perkin-Elmer Lambda 950 equipped with an integration sphere setup. SEM images were recorded using a Zeiss Ultra Plus field emission scanning electron microscope equipped with a GEMINI e-Beam column operated at $2 \mathrm{kV}$ with an aperture size set to $30 \mu \mathrm{m}$ to avoid excessive charging and radiation damage of the areas imaged. EDX spectroscopy was conducted with an X-Max $50 \mathrm{~mm}^{2}$ detector from Oxford Instruments operated at $10 \mathrm{kV}$. VSM measurements were performed at $300 \mathrm{~K}$ with a VSM (Quantum Design VersaLabTM 3T, Cryogen-free Vibrating Sample Magnetometer), cycling the applied field from -30 to +30 kOe two times with a step rate of $50 \mathrm{Oe} \mathrm{s}^{-1}$. Detailed analyses were carried out by cycling the applied field from -3 to $+3 \mathrm{kOe}$ at $5 \mathrm{Oe} \mathrm{s}^{-1}$. 


\section{Synthetic Procedures}

The synthesis of the iron oxide nanoparticles is based on a published procedure. ${ }^{23,29}$ Compounds DPP-1, ${ }^{30}$ TAPP, ${ }^{31}$ and DPP-TAPP-COF ${ }^{15}$ have been synthesized according to previously reported procedures.

\section{Iron Oxide Nanoparticles (FeO)}

For the precipitation of the iron oxide nanoparticles, $\mathrm{FeCl}_{3} \cdot 6 \mathrm{H}_{2} \mathrm{O}(8.0 \mathrm{mmol})$ and $\mathrm{FeCl}_{2} \cdot 4 \mathrm{H}_{2} \mathrm{O}(4.0 \mathrm{mmol})$ were dissolved in deionized $\mathrm{H}_{2} \mathrm{O}(100 \mathrm{~mL})$. Aqueous ammonia solution $(8 \mathrm{~mL})$ was quickly added with stirring. The black precipitate, which formed, was separated with a permanent handheld magnet after 1 minute and washed with $\mathrm{H}_{2} \mathrm{O}$ $(4 \times 100 \mathrm{~mL})$.

\section{$\mathrm{COOH}$ Functionalization of Iron Oxide Nanoparticles (COOH-FeO)}

The iron oxide nanoparticles were redispersed in citric acid $(50 \mathrm{~mL}, 0.01 \mathrm{M})$ and stirred for 30 minutes. The dispersion was destabilized by adding sodium hydroxide $(100 \mathrm{~mL}, 1.5 \mathrm{M})$, magnetically separated, and washed four times with $4: 1 \mathrm{EtOH} / \mathrm{H}_{2} \mathrm{O}(200 \mathrm{~mL})$. The precipitate was redispersed in water. The individual particles were inseparable; a stable ferrofluid was obtained. The $\mathrm{COOH}$-functionalized $\mathbf{C O O H}-\mathbf{F e O}$ nanoparticles were separated by centrifugation and redispersed in ${ }^{t} \mathrm{BuOH}$.

\section{$\mathrm{NH}_{2}$ functionalization of Iron Oxide Nanoparticles $\left(\mathrm{NH}_{2}-\mathrm{FeO}\right)$}

For the $\mathrm{NH}_{2}$ functionalization, unmodified aqueous iron oxide nanoparticle dispersion ( $45 \mathrm{~g}, 2 \mathrm{wt} \%$ ) was added to a three-necked flask. The dispersion was diluted with EtOH (22.5 g). 3-Amino-propyltriethoxysilane $(0.25 \mathrm{~g})$ was diluted with EtOH $(22.5 \mathrm{~g})$ and added within 10 minutes while stirring. Subsequently, the reaction flask was refluxed at $78^{\circ} \mathrm{C}$ for 3 hours whilst stirring. To remove all unreacted silane, the particles were magnetically separated and washed with $\mathrm{EtOH}$ for three times. Finally, the $\mathbf{N H}_{\mathbf{2}} \mathbf{-} \mathbf{F e O}$ nanoparticles were separated by centrifugation and redispersed in ${ }^{t} \mathrm{BuOH}$.

\section{FeO@DPP-TAPP-COF/POP Composite Materials}

In a Pyrex tube with screw cap, DPP-1 (11.6 mg, $0.02 \mathrm{mmol}$ ) and TAPP (6.7 $\mathrm{mg}, 0.01 \mathrm{mmol})$ were dissolved in ${ }^{n} \mathrm{BuOH}$ and mesitylene. To this mixture, an aliquot of a presonicated dispersion of the corresponding functional- ized nanoparticles $\mathbf{N H}_{\mathbf{2}}$ - $\mathbf{F e O}$ or $\mathbf{C O O H}-\mathbf{F e O}$ ( $4 \mathrm{wt} \%$ in ${ }^{t} \mathrm{BuOH}$, see Tables S1 and S2) was added. After the addition of acetic acid $(100 \mu \mathrm{L}, 6 \mathrm{M})$, the tube was sealed, sonicated for 5 minutes and heated at $120{ }^{\circ} \mathrm{C}$ for 5 days. The reaction product was filtered, washed several times with anhydrous THF, and further purified by solvent exchange with ethanol and acetone. To ensure the collection of composite materials, the magnetic fractions were attracted with a magnet prior to the solvent removal during this process. The obtained hybrid material was dried under high vacuum for 4 hours to obtain the composite materials as a black powder that shows magnetic properties.

\section{Funding Information}

Financial support by the Bavarian State Ministry of Science and the Arts in the Collaborative Research Network "Solar Technologies go Hybrid" is gratefully acknowledged. K.M. gratefully acknowledges funding by the DFG through grant MA 7252/4-2.

\section{Acknowledgment}

We thank Tim Granath (JMU Würzburg, FAU Erlangen) for the preparation of the nanoparticles and Maximilian Oppmann (Fraunhofer ISC, Würzburg) for VSM measurements. This publication was supported by the Open Access Publication Fund of the University of Würzburg.

\section{Supporting Information}

Supporting information for this article is available online at https://doi.org/10.1055/s-0040-1722655.

\section{References}

(1) (a) Sanchez, C.; Belleville, P.; Popall, M.; Nicole, L. Chem. Soc. Rev. 2011, 40, 696. (b) Faustini, M.; Nicole, L.; Ruiz-Hitzky, E.; Sanchez, C. Adv. Funct. Mater. 2018, 28, 1704158.

(2) Gower, L. B. Chem. Rev. 2008, 108, 4551.

(3) Mann, S. Nature 1993, 365, 499.

(4) Müller-Buschbaum, K.; Beuerle, F.; Feldmann, C. Microporous Mesoporous Mater. 2015, 216, 171.

(5) Mandel, K.; Granath, T.; Wehner, T.; Rey, M.; Stracke, W.; Vogel, N.; Sextl, G.; Müller-Buschbaum, K. ACS Nano 2017, 11, 779.

(6) Seuffert, M. T.; Wintzheimer, S.; Oppmann, M.; Granath, T.; Prieschl, J.; Alrefai, A.; Holdt, H.-J.; Müller-Buschbaum, K.; Mandel, K. J. Mater. Chem. C 2020, 8, 16010.

(7) Wintzheimer, S.; Reichstein, J.; Wenderoth, S.; Hasselmann, S.; Oppmann, M.; Seuffert, M. T.; Müller-Buschbaum, K.; Mandel, K. Adv. Funct. Mater. 2019, 29, 1901193.

(8) (a) Wehner, T.; Mandel, K.; Schneider, M.; Sextl, G.; MüllerBuschbaum, K. ACS Appl. Mater. Interfaces 2016, 8, 5445. (b) Wehner, 
T.; Seuffert, M. T.; Sorg, J. R.; Schneider, M.; Mandel, K.; Sextl, G.; Müller-Buschbaum, K. J. Mater. Chem. C 2017, 5, 10133.

(9) (a) Beuerle, F.; Gole, B. Angew. Chem. Int. Ed. 2018, 57, 4850. (b) Bisbey, R. P.; Dichtel, W. R. ACS Cent. Sci. 2017, 3, 533. (c) Chen, X.; Geng, K.; Liu, R.; Tan, K. T.; Gong, Y.; Li, Z.; Tao, S.; Jiang, Q.; Jiang, D. Angew. Chem. Int. Ed. 2020, 59, 5050.

(10) Ding, S.-Y.; Wang, W. Chem. Soc. Rev. 2013, 42, 548.

(11) (a) Rogge, S. M. J.; Bavykina, A.; Hajek, J.; Garcia, H.; OlivosSuarez, A. I.; Sepúlveda-Escribano, A.; Vimont, A.; Clet, G.; Bazin, P.; Kapteijn, F.; Daturi, M.; Ramos-Fernandez, E. V.; Llabrés, I. Chem. Soc. Rev. 2017, 46, 3134. (b) Pachfule, P.; Acharjya, A.; Roeser, J.; Langenhahn, T.; Schwarze, M.; Schomäcker, R.; Thomas, A.; Schmidt, J. J. Am. Chem. Soc. 2018, 140, 1423. (c) Wang, X.; Chen, L.; Chong, S. Y.; Little, M. A.; Wu, Y.; Zhu, W.-H.; Clowes, R.; Yan, Y.; Zwijnenburg, M. A.; Sprick, R. S.; Cooper, A. I. Nat. Chem. 2018, 10, 1180.

(12) Dogru, M.; Bein, T. Chem. Commun. 2014, 50, 5531.

(13) Mandal, A. K.; Mahmood, J.; Baek, J.-B. ChemNanoMat 2017, 3, 373.

(14) (a) Grzybowski, M.; Gryko, D. T. Adv. Opt. Mater. 2015, 3, 280. (b) Stolte, M.; Suraru, S.-L.; Diemer, P.; He, T.; Burschka, C.; Zschieschang, U.; Klauk, H.; Würthner, F. Adv. Funct. Mater. 2016, 26, 7415. (c) Soberats, B.; Hecht, M.; Würthner, F. Angew. Chem. Int. Ed. 2017, 56, 10771. (d) Liu, Q.; Bottle, S. E.; Sonar, P. Adv. Mater. 2020, 32, 1903882.

(15) Gole, B.; Stepanenko, V.; Rager, S.; Grüne, M.; Medina, D. D.; Bein, T.; Würthner, F.; Beuerle, F. Angew. Chem. Int. Ed. 2018, 57, 846.

(16) Rager, S.; Jakowetz, A. C.; Gole, B.; Beuerle, F.; Medina, D. D.; Bein, T. Chem. Mater. 2019, 31, 2707.

(17) (a) Pachfule, P.; Kandambeth, S.; Díaz Díaz, D.; Banerjee, R. Chem. Commun. 2014, 50, 3169. (b) Banerjee, T.; Gottschling, K.; Savasci, G.; Ochsenfeld, C.; Lotsch, B. V. ACS Energy Lett. 2018, 3 , 400. (c) Bhadra, M.; Kandambeth, S.; Sahoo, M. K.; Addicoat, M.; Balaraman, E.; Banerjee, R. J. Am. Chem. Soc. 2019, 141, 6152.

(18) (a) Stegbauer, L.; Schwinghammer, K.; Lotsch, B. V. Chem. Sci. 2014, 5, 2789. (b) Mullangi, D.; Dhavale, V.; Shalini, S.; Nandi, S.; Collins, S.; Woo, T.; Kurungot, S.; Vaidhyanathan, R. Adv. Energy Mater. 2016, 6, 1600110 .

(19) (a) Liao, Y.; Li, J.; Thomas, A. ACS Macro Lett. 2017, 6, 1444. (b) He, S.; Zeng, T.; Wang, S.; Niu, H.; Cai, Y. ACS Appl. Mater. Interfaces
2017, 9, 2959. (c) Romero, V.; Fernandes, S. P. S.; RodriguezLorenzo, L.; Kolen'ko, Y. V.; Espiña, B.; Salonen, L. M. Nanoscale 2019, 11, 6072.

(20) (a) Lin, G.; Gao, C.; Zheng, Q.; Lei, Z.; Geng, H.; Lin, Z.; Yang, H.; Cai, Z. Chem. Commun. 2017, 53, 3649. (b) Li, Y.; Zhang, H.; Chen, Y.; Huang, L.; Lin, Z.; Cai, Z. ACS Appl. Mater. Interfaces 2019, 11, 22492. (c) Wu, Y.; Sun, N.; Deng, C. ACS Appl. Mater. Interfaces 2020, 12, 9814. (d) Huang, L.; Mao, N.; Yan, Q.; Zhang, D.; Shuai, Q. ACS Appl. Nano Mater. 2020, 3, 319.

(21) (a) Wang, M.; Gao, M.; Zhang, K.; Wang, L.; Wang, W.; Fu, Q.; Xia, Z.; Gao, D. Mikrochim. Acta 2019, 186, 827. (b) Deng, Z.-H.; Wang, X.; Wang, X.-L.; Gao, C.-L.; Dong, L.; Wang, M.-L.; Zhao, R.-S. Mikrochim. Acta 2019, 186, 108.

(22) Tan, J.; Namuangruk, S.; Kong, W.; Kungwan, N.; Guo, J.; Wang, C. Angew. Chem. Int. Ed. 2016, 55, 13979.

(23) Mandel, K.; Straßer, M.; Granath, T.; Dembski, S.; Sextl, G. Chem. Commun. 2015, 51, 2863.

(24) (a) Mandel, K.; Hutter, F.; Gellermann, C.; Sextl, G. ACS Appl. Mater. Interfaces 2012, 4, 5633. (b) Mandel, K.; Hutter, F. Gellermann, C.; Sextl, G. J. Magn. Magn. Mater. 2013, 331, 269. (c) Brede, F. A.; Mandel, K.; Schneider, M.; Sextl, G.; MüllerBuschbaum, K. Chem. Commun. 2015, 51, 8687. (d) Müssig, S.; Fidler, F.; Haddad, D.; Hiller, K. H.; Wintzheimer, S.; Mandel, K. Adv. Mater. Technol. 2019, 4, 1900300.

(25) Wei, Y.; Han, B.; Hu, X.; Lin, Y.; Wang, X.; Deng, X. Procedia Eng. 2012, 27, 632.

(26) Keller, N.; Calik, M.; Sharapa, D.; Soni, H. R.; Zehetmaier, P. M.; Rager, S.; Auras, F.; Jakowetz, A. C.; Görling, A.; Clark, T.; Bein, T.J. Am. Chem. Soc. 2018, 140, 16544.

(27) Bucher, L.; Tanguy, L.; Fortin, D.; Desbois, N.; Harvey, P. D.; Sharma, G. D.; Gros, C. P. ChemPlusChem 2017, 82, 625.

(28) (a) Long, B.; Bakr, O.; Stellacci, F. J. Exp. Nanosci. 2008, 3, 53. (b) Zanetti-Polzi, L.; Amadei, A.; Djemili, R.; Durot, S.; Schoepff, L.; Heitz, V.; Ventura, B.; Daidone, I.J. Phys. Chem. C 2019, 123, 13094.

(29) Mandel, K.; Hutter, F.; Gellermann, C.; Sextl, G. Colloids Surf., A 2011, 390, 173.

(30) Huo, L.; Hou, J.; Chen, H.-Y.; Zhang, S.; Jiang, Y.; Chen, T. L.; Yang, Y. Macromolecules 2009, 42, 6564.

(31) Meng, S.; Xu, Z.; Hong, G.; Zhao, L.; Zhao, Z.; Guo, J.; Ji, H.; Liu, T. Eur. J. Med. Chem. 2015, 92, 35. 